\title{
Filamentation and coalescence of singular optical pulses in narrow-gap semiconductors and modeling of self-organization of vortex solitons using two-photon absorption
}

\author{
V. Skarka, ${ }^{1}$ N. B. Aleksić, ${ }^{2}$ M. Derbazi, ${ }^{1}$ and V. I. Berezhiani ${ }^{3}$ \\ ${ }^{1}$ Laboratoire POMA, CNRS FRE 2988, Université d'Angers, 2 Boulevard Lavoisier, 49045 Angers Cedex 1, France \\ ${ }^{2}$ Institute of Physics, Pregrevica 118, 11000 Belgrade, Serbia \\ ${ }^{3}$ Andronikashvili Institute of Physics and Javakhishvili Tbilisi State University, Tbilisi, Georgia
}

(Received 5 March 2009; revised manuscript received 4 December 2009; published 12 January 2010)

\begin{abstract}
Short intense laser pulses with phase singularity propagating in narrow-gap semiconductors are modeled. The saturating nonlinearity is a prerequisite for self-organization of pulses into solitons. The cubic-quintic saturation appears due to the conduction-band nonparabolicity in synergy with the free carriers excitation through two-photon absorption. The pulse stability analyzed using Lyapunov's method is confirmed by numerical simulations. Depending of its power, a singular Gaussian pulse far from equilibrium either filaments or subsequently coalesces evolving toward vortex soliton. Above breaking power, such a vortex soliton resists to azimuthal symmetry-breaking perturbations.
\end{abstract}

DOI: 10.1103/PhysRevB.81.035202

PACS number(s): 42.70.Nq, 42.65.Tg

\section{INTRODUCTION}

New developments in the generation of femtosecond laser pulses propagating in promising nonlinear materials have brought a wide variety of new phenomena within the realm of investigation. Self-guided optical solitary waves have received much attention in connection with possible applications in all-optical signal processing and laser systems. ${ }^{1}$ Spatiotemporal solitons completely localized in space and in time are called light bullets. Such bright solitons are stable whenever the diffraction and dispersion are compensated by saturating nonlinearity. Indeed, multidimensional solitons in media with only cubic nonlinearity are subject to the collapse. The collapse is prevented in a material (if any) containing also the quintic nonlinearity of opposite sign. ${ }^{2}$ Highpower nodeless beams illuminating an appropriate phase mask yield to vortex states that propagate through long distances. ${ }^{3}$ Vortex solitons are robust steady-state structures with phase singularity and nonzero angular momentum. Selfdefocusing media support optical vortex solitons (OVSs) corresponding to a zero intensity center surrounded by a bright infinite background. ${ }^{4}$ Localized optical vortex solitons (LOVSs) with phase singularity surrounded by one or few bright rings are generated in self-focusing media. The phase change around the vortex presents an integer number $m$ of windings called the topological charge of the vortex. This topological charge is proportional to the angular momentum that can be large. ${ }^{5}$ In self-defocusing Kerr media the OVS with $m=1$ is stable for small perturbation. ${ }^{6}$ In self-focusing media, ordinary saturating nonlinearities make the LOVS stable against radial perturbation, but do not prevent azimuthal instabilities. The initial ring breaks into filaments flying off tangentially. The ring breakup is observed experimentally in sodium vapor. ${ }^{7}$ Materials with the saturating nonlinearity, in particular the very promising cubic-quintic ones, have been searched for long time. The reason is the LOVS stability in cubic-quintic media for high intensity in spite of the break for low one. ${ }^{8}$ Indeed, for small intensities of the beam, the medium is self-focusing since the derivative of the nonlinear index of refraction $\delta n$ with respect to the laser beam intensity $I$ is positive. The medium becomes selfdefocusing $(d \delta n / d I<0)$ with increase of field intensity. Therefore, the laser beam is defocusing at the maximum field intensity while it is self-focusing at its wings. If the bulk of LOVS is self-defocusing, it behaves like OVS and becomes stable. Thus, we demonstrated that cubic-quintic media support both stable LOVS and OVS allowing applications in optical switching. ${ }^{9}$ Competing cubic-quintic nonlinearity can be used for spatial solitons dual-channel trapping in planar waveguide too. ${ }^{10}$ Vortex soliton structures can be expected in diverse types of focusing-defocusing media. For instance, a self-focusing electromagnetic field is saturated inducing the ionization of air. As a consequence, vortex solitons are self-organized. ${ }^{11}$ Same solitonic mechanism seems to be involved in the process of lightning. ${ }^{12}$ Recently, atmospheric propagation of spinning femtosecond light pulses has been studied using $(2+1)$-dimensional model of multiphoton ionization and higher vortex charges, together with threedimensional (3D) numerical simulations. ${ }^{13,14}$ Vortex solitons are stable also in a vectorial cubic-quintic model. ${ }^{15}$ The vortex lattices can be generated numerically in such media. ${ }^{16}$ In order to find in practice media with cubic-quintic nonlinearity, we investigated semiconductors.

Semiconductors are one of the most promising classes of nonlinear media since they have, in comparison to most other nonlinear materials, larger nonlinearities and an extremely short response time. ${ }^{17}$ Variety of reactive and active mechanisms can generate refractive index nonlinearities in different kinds of semiconductors. Narrow gap semiconductors, in particular the III-V compounds with a small effective mass of free equilibrium conduction electrons, exhibit a large degree of nonparabolicity of the electronic conduction band. In the Kane's model, Hamiltonian of a conduction-band electron is written in the form

$$
\mathbf{H}=\frac{\mathbf{E}_{g}}{2}\left(1+\frac{p^{2}}{m_{*}^{2} c_{*}^{2}}\right)^{1 / 2},
$$

where $p$ is a quasimomentum and $\mathbf{E}_{g}$ is the forbidden gap width separating valence and conduction bands. ${ }^{18}$ Here, 
$c_{*}=\left(\mathbf{E}_{g} / 2 m_{*}\right)^{1 / 2}$ plays the part of the speed of light $\left(c_{*} \approx 3.7 \times 10^{-3} c\right.$ for $\left.\mathrm{InSb}\right)$ and $m_{*}$ is the effective mass of electrons at the bottom of the conduction band.

3 decades ago, it has been demonstrated that a weak nonparabolicity in InSb can lead to the self-focusing of the $\mathrm{CO}_{2}$ laser beam. ${ }^{19}$ At that time, laser beam nonlinear interactions with semiconductors have been studied mainly on a nanosecond time scale. Consequently, in order to avoid the breakdown of the semiconductor for field intensity of $10^{7} \mathrm{~W} / \mathrm{cm}^{2}$, the nonparabolicity factor $p^{2} / m_{*}^{2} c_{*}^{2} \sim e^{2} E^{2} / m_{*}^{2} c_{*}^{2} \omega^{2}$ had to be kept much lower than unity. $E$ and $\omega$ are, respectively, laser electric field and its frequency. Furthermore, the comparatively small collision time of carriers, typically $\tau_{R} \approx 10^{-12}-10^{-13} \mathrm{~s}$, could hinder the excitation of collective modes in semiconductor plasma. ${ }^{20}$ Due to these limitations, expected nonlinear effects appeared to be small and therefore the research in this direction has not been continued.

Due to current technology, it is possible to produce femtosecond pulses of high intensity with wavelengths ranging from the ultraviolet to the midinfrared. Thus, available pulses are much shorter than the breaking time (about $100 \mathrm{ps}$ ), allowing us to consider effects with applied intensities as high as few $\mathrm{GW} / \mathrm{cm}^{2}$ and nonparabolicity factor close to 1 . However, the pump fluency should be kept below $0.5 \mathrm{~kJ} / \mathrm{m}^{2}$ in order to avoid the semiconductor melting even on a subpicosecond time scale. ${ }^{21}$ Achievements in short-pulse generation have motivated new studies of such pulse propagation in the plasma of narrow-gap $n$-doped semiconductors with the conduction-band nonparabolicity generating the nonlinear refraction. $^{22}$ Furthermore, the modulation instability in narrow-gap semiconductors is suggested to produce subpicosecond midinfrared pulses. ${ }^{23}$

In this paper, we investigate, using analytical and numerical methods, the dynamics of short singular pulses in the narrow-gap semiconductors exhibiting not only reactive but also active mechanisms related to the energy dissipation of laser radiation. Both mechanisms in synergy generate a saturating refractive nonlinearity. Indeed, a strong nonparabolicity of InSb due to a very narrow gap $\left(\mathbf{E}_{g}=0.234 \mathrm{eV}\right.$ at $T=77 \mathrm{~K}$ ) induces an important third-order nonlinear susceptibility. Simultaneously, laser radiation excites nonequilibrium free carriers through two-photon absorption. As a consequence, a nonlinear susceptibility of the fifth order is generated through an active mechanism. ${ }^{17}$ The nonlinearity relaxation time depends on the type of nonequilibrium carriers recombination. The linear recombination through impurities $\left(\tau_{R} \approx 10^{-3}-10^{-8} \mathrm{~s}\right)$, the square recombination $\left(\tau_{R} \approx 10^{-8}-10^{-9} \mathrm{~s}\right)$, as well as the cubic one (less than $10^{-9} \mathrm{~s}$ in the case of high nonequilibrium carrier concentrations) take all much longer time than the pulse temporal width. The nonlinearity involved by the excitation of nonequilibrium free carriers is present in diverse types of semiconductors (e.g., Si, $\mathrm{Hg}_{1-x} \mathrm{Cd}_{x} \mathrm{Te}, \mathrm{ZnO}, \mathrm{CdS}, \mathrm{CdS}_{x} \mathrm{Se}_{1-x}$, $\mathrm{CdSe}, \mathrm{CdTe}$, and $\mathrm{ZnSe}) .{ }^{17} \mathrm{In}$ the bulk semiconductors which exhibit abovementioned cubic-quintic saturation nonlinearity, we investigate the conditions for a balance between nonlinear focusing and defocusing resulting in a self-guided multidimensional vortex soliton. The evolution of singular pulses, leading either to the filamentation or to the coalescence as well as the self-organization of stable soliton, is modeled. A stability criterion, based on variation method, is established giving conditions for self-organization into LOVS.

\section{VORTEX SOLITONS AS STABLE SINGULAR PULSES}

Let us search for mechanisms leading to the cubic-quintic nonlinearity in semiconductor media. The evolution of a singular pulse in semiconductors is governed by the nonlinear Schrödinger (NLS) equation in commoving coordinates, $\tau=t-\zeta / v_{g}$,

$$
2 i k \frac{\partial \mathcal{E}}{\partial \zeta}+\Delta \mathcal{E}+2 k^{2} \frac{\delta n}{n_{0}} \mathcal{E}=0,
$$

where $\mathcal{E}$ is a slowly varying field envelope, $v_{g}=d \omega / d k$ is the group velocity, and $k=k_{o} n_{o}$ the wave vector. ${ }^{22}$ The Laplacian $\Delta \mathcal{E}$ describes beam diffraction and anomalous group-velocity dispersion, while $n_{0}$ and $\delta n\left(|\mathcal{E}|^{2}\right)$ are, respectively, linear and nonlinear optical indices. Two dominating nonlinear effects contributing to the nonlinear refractive index $\delta n=n_{2}|\mathcal{E}|^{2}+n_{4}|\mathcal{E}|^{4}$ are, respectively, the nonparabolicity of conduction band and the excitation of nonequilibrium free carriers through two-photon absorption. In weakly doped semiconductors with free-electron concentration $N \approx 2.14 \times 10^{16} \mathrm{~cm}^{-3}$ (e.g., at the temperature $T=77 \mathrm{~K}$ ), the nonparabolicity generates the nonlinear refractive index $n_{2}$ written in simplified form ${ }^{17,24}$

$$
n_{2}=\frac{\pi N_{2} e^{4}}{2 n_{0} m_{*}^{2} \mathbf{E}_{g} \omega^{4}}\left(1+\frac{6 K_{B} T}{\mathbf{E}_{g}}\right)^{-7 / 2},
$$

where $K_{B}$ stands for Boltzmann constant.

The time variation of nonequilibrium free carriers $N_{2}$ excited through two-photon absorption reads

$$
\frac{d N_{2}}{d t}=\frac{\alpha_{2} I^{2}}{2 \hbar \omega},
$$

where $\alpha_{2}$ is two-photon absorption coefficient and the laser field intensity $I=c n_{0}|\mathcal{E}|^{2} / 8 \pi$. The linear, square, and cubic recombinations are all neglected in Eq. (4) since much longer than the chosen pulse temporal width $\tau_{p}=200 \mathrm{fs}$. Substituting integrated Eq. (4) $N_{2}=\alpha_{2} / 2 \hbar \omega \int_{-\infty}^{\tau} I^{2} d \tau$ into Eq. (2) leads to the complicate integrodifferential equation that governs the evolution of laser field envelope. For shortdistance propagation, this time integration gives approximately a constant $\tau_{0}{ }^{25}$ Such a freezing of free carriers over pulse duration under considered conditions should not significantly alter the spatial dynamics. This simplification is justified taking into account the short-distance propagation in semiconductors as can be seen from characteristic values obtained below for chosen laser and semiconductor parameters. In this case, the electron density is approximated by the expression $N_{2}=\alpha_{2} \tau_{0} n_{0}^{2} c^{2}|\mathcal{E}|^{4} / 64 \pi^{2} \hbar \omega$, where $\tau_{0}=\tau_{p} \sqrt{\pi / 2}$ is related to the laser-pulse temporal width $\tau_{p}$ (half width at half maximum). As a consequence, from the classical DrudeLorentz formula determining the contribution of electronhole plasma to dielectric constant, the nonlinear refractive index $n_{4}$ induced through two-photon excitation of nonequilibrium free carriers can be expressed as 


$$
n_{4}=-\frac{\alpha_{2} \tau_{0} n_{0} e^{2}}{64 \pi^{2} \hbar \omega^{3} m_{*}},
$$

where $\hbar$ is Planck constant. ${ }^{17}$

In Eq. (2), we neglect effects related to the laser-pulse reshaping due to the group-velocity dispersion since in moderately dense semiconductor plasma, the group-velocity dispersion is small provided laser-pulse duration no shorter than 100 fs. Therefore, the NLS is simplified

$$
i \frac{\partial \mathcal{E}}{\partial \zeta}+\frac{1}{2 k} \Delta_{\perp} \mathcal{E}+k_{o}\left(n_{2}|\mathcal{E}|^{2}+n_{4}|\mathcal{E}|^{4}\right) \mathcal{E}=0
$$

where the transverse Laplacian $\Delta_{\perp} \mathcal{E}=\partial^{2} \mathcal{E} / \partial x^{2}+\partial^{2} \mathcal{E} / \partial y^{2}$ takes into account separately two transverse coordinates $x$ and $y$ allowing the consideration of asymmetric inputs usual in experiments. Thus, we get the NLS with saturating nonlinearity due to the synergy of two dominating effects: the nonparabolicity and the excitation of free carriers through two-photon absorption. Particularity of this kind of saturating nonlinearity is that nonlinear index of refraction is focusing at lower intensities of the beam but becomes defocusing at higher intensities. Indeed, if the pick intensity of the laser $I_{m}$ is larger than the critical intensity $I_{c}=n_{o} c n_{2} /\left(16 \pi\left|n_{4}\right|\right)$, the nonlinear medium acts as self-defocusing near the pick of the beam while it remains focusing at the beam wings. Let us estimate the critical intensity $I_{c}$ for real experimental conditions. Typical values are for the gap $\mathbf{E}_{g}=0.234 \mathrm{eV}$ and for the effective mass $m_{*}=1.518 \times 10^{-32^{\circ}} \mathrm{kg}$. For the laser wavelength $\lambda=10.6 \mu \mathrm{m}$, the two-photon absorption coefficient is $\alpha_{2}=8 \mathrm{~cm} / \mathrm{MW} .{ }^{26}$ For instance, for the laser-pulse temporal width $\tau_{p}=200 \mathrm{fs}$, the critical intensity is $I_{c} \approx 72.47 \mathrm{MW} / \mathrm{cm}^{2}$. Dimensionless variables can be introduced in Eq. (6) using characteristic values for normalization: $\quad z=\zeta / Z, \quad$ where $Z=2 k_{o} n_{o} R^{2}, \quad r_{\perp}=\rho / R$, with $R=\sqrt{\left|n_{4}\right| / n_{o}} /\left(k_{o} n_{2}\right) \approx 917 \mu \mathrm{m}, \quad$ and $E=\mathcal{E} / \mathcal{E}_{c}$, where $\mathcal{E}_{c}=\sqrt{n_{2} / 2\left|n_{4}\right|}$. The dimensionless NLS reads

$$
i \frac{\partial E}{\partial z}+\Delta_{\perp} E+\left(|E|^{2}-\frac{1}{2}|E|^{4}\right) E=0 .
$$

This equation reduces to an ordinary differential equation assuming solutions in polar coordinates $E=A_{o}(r) \exp (i m \theta+i \beta z)$ with an amplitude $A_{o}$ and a propagation constant $\beta .{ }^{9}$ Considering only the lowest-order radial eigenmode for the topological charge $m=1$, numerical simulations under well-defined conditions yield localized solitary solutions. The azimuthal modulation instability breaks vortex rings with topological charges of higher order. ${ }^{8,13,14}$ The solitary mode power $P=\int d \mathbf{r}_{\perp}|E|^{2}$ is a growing function of $\beta$ (see Fig. 1) since the maximum field amplitude $A$ increases with $\beta$ (as in Fig. 2). For $A \rightarrow 0$, the equilibrium power approaches to the critical power $P_{c} \approx 50.3$, i.e., the minimum power necessary for compensation of diffraction by selffocusing. Consequently, the necessary condition to generate a LOVS is that the laser beam power exceeds $P_{c}$. Such a power is about 4 times larger than the critical one needed for the generation of fundamental solitons with zero topological charge. ${ }^{27}$ The amplitude slope $S$ is growing until the propagation constant critical value $\beta_{c}=0.3$, while it is decreasing

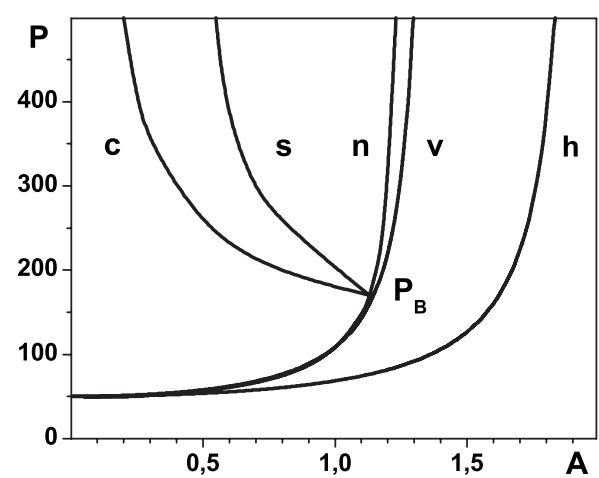

FIG. 1. The variation $(v)$ and numerical $(n)$ curves of power $P$ vs amplitude $A$ as well as coalescence $(c)$, stability $(s)$, and Hamiltonian $(h)$ curves.

for larger $\beta$. Above $\beta_{c}$, the normalized amplitude $A$ is larger than one implying self-defocusing near the laser beam maximum and self-focusing at the beam wings. With increase of $\beta$, the central part of the vortex soliton flattens since its bulk part is in the defocusing region. The linear stability analyses can be performed in order to check the stability of these stationary solutions. The field amplitude in Eq. (7) is increased by a small quantity $a^{+}(r, z) \exp (+i L \theta)$ $+a^{-}(r, z) \exp (-i L \theta)$ depending of the integer azimuthal index $L$. After linearization with respect to the perturbations, two coupled equations

$$
\begin{aligned}
& \left\{i \frac{\partial}{\partial z}+\frac{1}{r} \frac{\partial}{\partial r} r \frac{\partial}{\partial r}-\beta-\frac{(m \pm L)^{2}}{r^{2}}+2 A_{o}^{2}-3 A_{o}^{4} / 2\right\} a^{ \pm} \\
& +\left(A_{o}^{2}-A_{o}^{4} / 2\right)\left(a^{\mp}\right)^{*}=0
\end{aligned}
$$

are obtained.

The growth rate $\gamma$ as a function of the azimuthal index $L$ is given in Fig. 3 for different values of propagation constant $\beta$. The azimuthal periodicity involves integer $L$ although it appears in the linearized equation as a continuous parameter. ${ }^{9}$ The vortex soliton is stable for radial perturbations $(L=0)$ but not for azimuthal ones if the propagation constant $\beta<\beta_{c}$. Indeed, numerical simulations below the breaking power $P_{B} \approx 171$ (see Fig. 1) give evidences of a quickly developing breaking instability corresponding to the

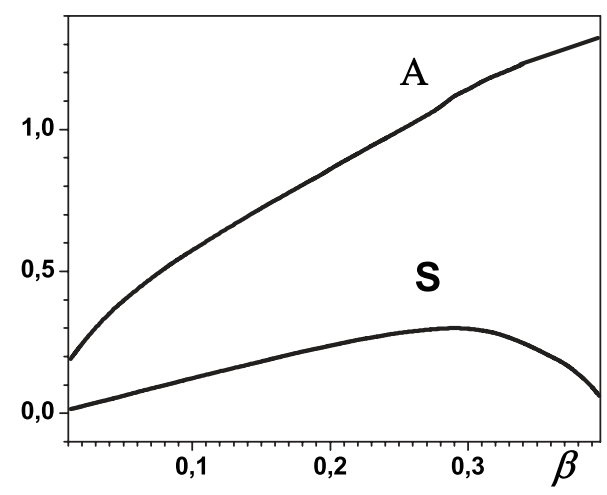

FIG. 2. The amplitude $A$ and the spatial derivative of the field at the origin $S$ vs the propagation constant $\beta$ for $m=1$. 


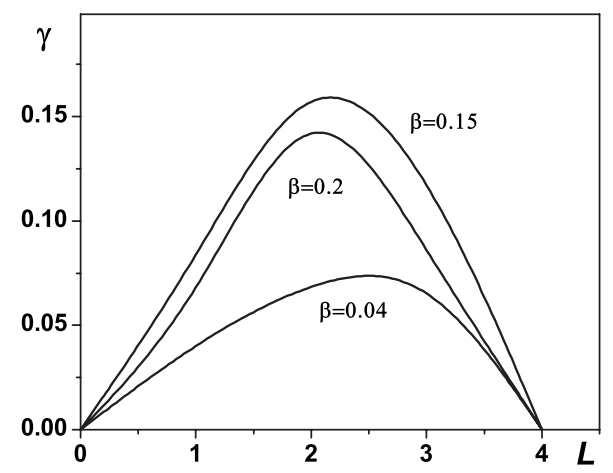

FIG. 3. Growth rate $\gamma$ as a function of the azimuthal index $L$ for propagation constants $\beta=0.04, \beta=0.15$, and $\beta=0.2$.

maximal growth rate $\Gamma>0$ in Fig. 4. The maximal growth rate in Fig. 3 corresponds roughly to the integer $L=2$. As a consequence, the beam breaks into two filaments, similar to spatial solitons, running away tangentially in opposite directions in order to conserve the total angular momentum $|M|=|m| P$ (as can be seen in Fig. 6). For $\beta$ larger than the critical one, maximal growth rate is zero (see Fig. 4). Indeed, numerical simulations give a stable vortex soliton.

\section{EVOLUTION AND STABILITY OF SINGULAR PULSES}

In experiments, a singular input pulse is usually generated far from equilibrium, thus, it differs substantially from the vortex soliton. The evolution of such a pulse is governed by nonintegrable Eq. (7) and can be followed exactly only by numerical simulations. Nevertheless, analytical approaches even though approximate are of great help in physical understanding and in order to give a hint for simulations. Integrals of motion conserved by the Eq. (7) are beam power, angular momentum, and Hamiltonian. The following inequality holds:

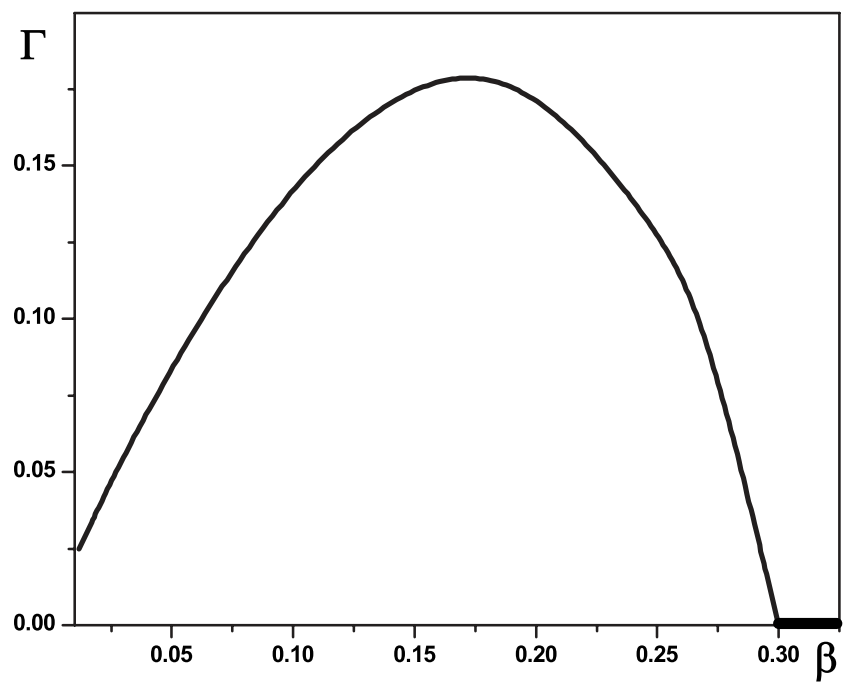

FIG. 4. Maximal growth rate $\Gamma$ as a function of the propagation constant $\beta$.

$$
H=\int d \mathbf{r}_{\perp}\left(\left|\nabla_{\perp} E\right|^{2}-|E|^{4} / 2+|E|^{6} / 6\right)=-1 / 6 \int d \mathbf{r}_{\perp}|E|^{6}<0
$$

in the self-trapping region on the left of the curve $h$ (in Fig. 1) corresponding to the zero Hamiltonian. Negativeness of the Hamiltonian prevents diffraction during evolution since the maximum field intensity has a lower bound $|E|_{\text {max }}^{2}>2|H| / P$ independent of the propagation variable $z \cdot{ }^{28}$ The saturating cubic-quintic nonlinearity in Eq. (7) prevents the wave collapse. As a consequence, neither azimuthal nor radial modulation instability does lead to either diffraction or collapse. Therefore, the pulse is self-trapped and strongly attracted to the equilibrium state corresponding to the vortex soliton. However, due to the azimuthal instability, the beam can break in multiple filaments before reaching the attractor.

In order to study rather complex dynamic properties of solutions of Eq. (7), numerical simulations are required. ${ }^{9}$ In a different context, numerical simulation of atmospheric propagation in $(2+1)$ - and $(3+1)$-dimensional cases have been performed in Refs. 11, 13, and 14. However, a better insight in nonlinear dynamics of a singular Gaussian beam can be obtained using the variational approach. Although it is unable to account structural changes of beam profile, this method can serve as a guideline for simulations. ${ }^{29}$ In general, $p$-photons absorption can be described by the generalized nonlinear Schrödinger (GNLS) equation with $(2 p+1)$-order saturating nonlinearity ${ }^{11,13,14}$

$$
i \frac{\partial E}{\partial z}+\Delta_{\perp} E+|E|^{2} E-\frac{1}{p}|E|^{2 p} E=0 .
$$

Experimental conditions often involve asymmetric inputs. Therefore, a trial function asymmetric with respect to transverse variables $x$ and $y$ is chosen

$$
\begin{aligned}
E= & A\left(\frac{e}{m}\right)^{m / 2}\left(\frac{x}{X}+i \frac{y}{Y}\right)^{m} \\
& \times \exp \left[-\frac{x^{2}}{2 X^{2}}-\frac{y^{2}}{2 Y^{2}}+i C x^{2}+i D y^{2}+i \psi\right] .
\end{aligned}
$$

Parameters to optimize are the maximum amplitude $A$, pulse widths $X$ and $Y$, wave-front curvatures $C$ and $D$, as well as phase $\psi$. Corresponding Euler-Lagrange equations are obtained by variation of the Lagrangian density (averaged over $x$ and $y$ ) with respect to amplitude ${ }^{30}$

$$
\frac{d A}{d z}=-2 A(C+D)=F_{A},
$$

widths

$$
\begin{aligned}
& \frac{d X}{d z}=4 X C=F_{X}, \\
& \frac{d Y}{d z}=4 Y D=F_{Y},
\end{aligned}
$$

curvatures 


$$
\begin{aligned}
& \frac{d C}{d z}=-4 C^{2}+\frac{1}{X^{4}}-\frac{1}{R^{2} X^{2}} \frac{A^{2}}{B^{2}}+\frac{1}{p R^{2} X^{2}} \frac{A^{2 p}}{B^{2 p}}=F_{C}, \\
& \frac{d D}{d z}=-4 D^{2}+\frac{1}{Y^{4}}-\frac{1}{R^{2} Y^{2}} \frac{A^{2}}{B^{2}}+\frac{1}{p R^{2} Y^{2}} \frac{A^{2 p}}{B^{2 p}}=F_{D},
\end{aligned}
$$

and phase

$$
\frac{d \psi}{d z}=-(m+1)\left(\frac{1}{X^{2}}+\frac{1}{Y^{2}}\right)+\frac{3}{R^{2} B^{2}} A^{2}-\frac{(2 p+1)}{p^{2} R^{2} B^{2 p}} A^{2 p}
$$

The amplitude is normalized by a factor $B$ $=2^{m+1 / p-1}(m / e)^{m / 2} w$ and the width by $R=2^{(m+1) p / p-1} w^{-1}[(m$ $+1) ! /(2 m) !]^{1 / 2}, \quad$ where $\quad w=\left(\left[(p+1)^{2 m(p+1)+2}(2 m) !\right]\{p[(p\right.$ $\left.+1) m] !\}^{-1}\right)^{1 / 2 p-2}$. Therefore, the GNLS Eq. (10) is reduced to the set of six ordinary differential equations (ODEs). In order to obtain corresponding steady-state solutions, right-hand side of each of Eqs. (12)-(16) denoted by $F$ with corresponding subscript is set to zero $F_{A}=F_{X}=F_{Y}=F_{C}=F_{D}=0 .{ }^{30}$ The solitonic equilibrium corresponds to the symmetric steady state. As a consequence, widths are equal $X=Y$ and wavefront curvatures are zero $D=C=0$. Conserved beam power $P=\pi m !\left(\frac{e}{m}\right)^{m} A^{2} X Y$ depends in the equilibrium only of the amplitude

$$
P=\frac{4^{(m+1)} \pi(m+1)(m !)^{2}}{(2 m) ! \sqrt{1-p^{-1}(A / B)^{2 p-2}}},
$$

as well as the width

$$
X=\frac{R}{\sqrt{(A / B)^{2}-p^{-1}(A / B)^{2 p}}} .
$$

In order to establish the stability criterion for these steady states, the Lyapunov's method is used. A Jacoby determinant is constructed from right-hand sides of Eqs. (12)-(16) differentiated with respect to widths $X$ and $Y$ (e.g., $\partial F_{S} / \partial Y$ ), curvatures $C$ and $S$ (e.g., $\partial F_{X} / \partial C$ ), and amplitude $A$ (e.g., $\left.\partial F_{A} / \partial A\right)$ in equilibrium. ${ }^{29,30}$ The steady-state solutions of five coupled ODE are stable if the real part of solutions of the equation $\left(\lambda^{2}+\alpha_{1}\right)\left(\lambda^{2}+\alpha_{2}\right) \lambda=0$ is negative. Following Hurwitz, this condition can be fulfilled if coefficients of this equation are positive. The first coefficient is always positive

$$
\alpha_{1}=\frac{8\left(A^{2} B^{2 p}-p^{-1} A^{2 p} B^{2}\right)^{2}}{R^{4} B^{4(p+1)}}>0 .
$$

Therefore, the stability criterion concerns only the second coefficient

$$
\alpha_{2}=\frac{8(p-1) A^{2 p}\left(A^{2} B^{2 p}-p^{-1} A^{2 p} B^{2}\right)}{p R^{4} B^{4 p+2}},
$$

which is positive only if the amplitude $A<A_{c}=B p^{1 / 2 p-2}$. In the two-photon case we considered here $(p=2$ and $m=1)$, equilibrium steady states are stable if the amplitude $A$ is less than the critical one $A_{c}=1.365$. In Fig. 1, variational curve $(v)$ and exact numerical one $(n)$ corresponding to vortex solitons coincide only for small power. For large power, the numerical curve is steeper allowing higher power for amplitudes close to the critical one. In general, the stability criterion [Eq. (21)] allows finding for multiphoton absorption stable solutions of GNLS corresponding to LOVS.

\section{NUMERICAL SIMULATIONS OF PULSE DYNAMICS}

Following the aboveobtained analytical and numerical results, the vortex soliton is stable to azimuthal perturbations only above breaking power $P_{B} \approx 171$. Below this power, the vortex soliton breaks into two filaments corresponding to stable bright solitons flying off tangentially. However, taking into account radiative losses during propagation, vortex solitons are self-organized only from pulses either close enough to the stable equilibrium or with input power well above the breaking one. Pulse dynamics simulations give for different input parameters a complex picture of various behaviors which near the equilibrium agree with analytically obtained results. On the right of the stability curve $s$ in Fig. 1, for the power well above the critical one, stable vortex structures are generated even before reaching soliton attractors. A similar behavior can be found also on the right of the equilibrium curve where the trapping zone reaches zero Hamiltonian curve $h$. Although the attraction to the equilibrium is exercised on both sides of the equilibrium curve, the pulse evolution is quite different. For a given power, the modulation instability occurs only for large pulse widths, thus, for small amplitudes. A singular Gaussian input beam of small amplitude is subject of filamentations due to the modulation instability. Following numerical simulations, a singular Gaussian input beam of high power $(P \approx 300-500)$ and small amplitudes $(A \approx 0.1-0.3)$, due to the rapidly developing modulation instability, breaks into a multitude of four filaments symmetrically distributed in order to ensure conservation of total angular momentum. For instance, the input ring in Fig. 5(a) with power $P=400$ and amplitude $A=0.26$ (on the coalescence curve $c$ in Fig. 1) splits into eight filaments $(b)$. Four filaments increase their intensity on the expenses of the remaining four, coalescing into a crosslike structure $(c)$. The coalescence is achieved in Fig. 5(d), attracting weaker filaments into a "froggy" rotating structure $(d)$. Weaker filaments are loosing their intensity flying off tangentially to the cross vortex $(e)$ and are vanishing soon $(f)$. Four remaining filaments split again flying away from the equilibrium state. Such a coalescence appearing in general in the triangular region between curves $c$ and $s$ seems to be related with the attraction that the equilibrium vortex soliton states exhibit against the modulation instability induced filamentation. However, the final issue of the coalesced vortex depends of its initial distance from the equilibrium curve $n$ and its power since during the evolution, the beam power diminishes due to radiative losses. More intense pulses closer to the equilibrium such as the one in Fig. 6(a) with power $P=350$ and amplitude $A=0.6$ will split into four filaments $(b)$ coalescing subsequently into a stable squarelike vortex structure $(c)$. Such a vortex exhibits damping oscillations around the equilibrium where it finally self-organizes into a vortex soliton 


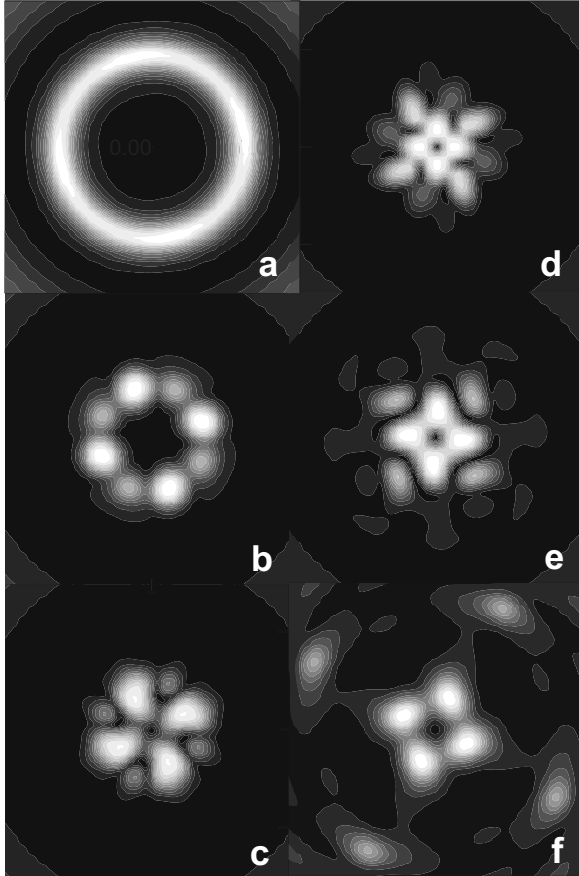

FIG. 5. (a) Pulse splitting into eight filaments for (b) $z=95$, coalescence from (c) $z=100$ until (e) $z=110$, and (f) breaking into four filaments at $z=130$.

(d). Less-powerful pulses, for instance, the one with power $P=250$ and amplitude $A=0.5$ (in Fig. 7), will first split into four twisting filaments, coalescing twice before reaching unstable equilibrium below breaking power $P_{B} \approx 171$. As a consequence, the vortex after second coalescence, instead to be stabilized into a vortex soliton, breaks into two solitonic filaments flying off tangentially to the initial ring.

In conclusion, we proposed optical materials displaying cubic-quintic nonlinearity which has been searched for long time. Indeed, we demonstrated that singular laser pulses can

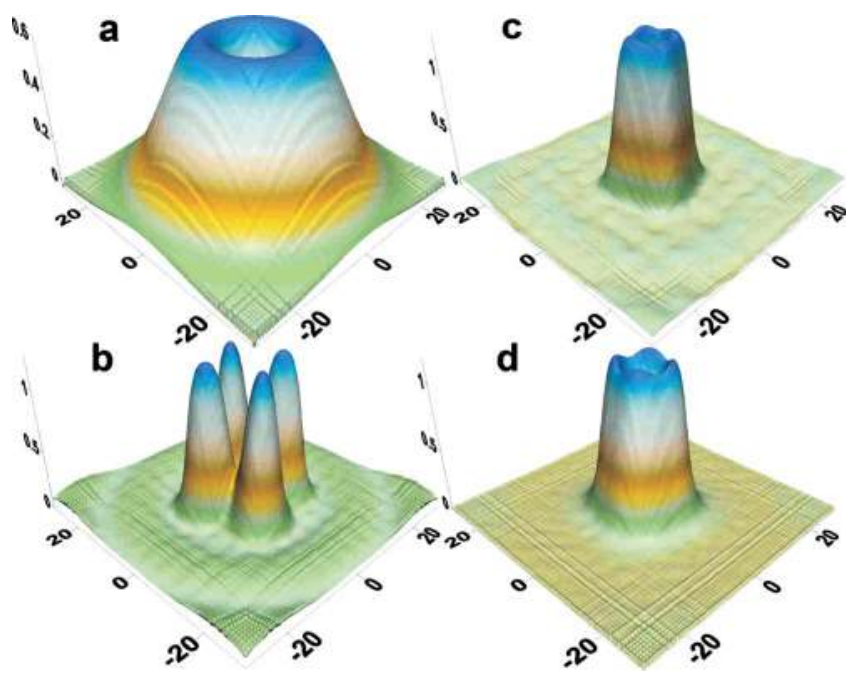

FIG. 6. (Color online) (a) Input pulse at $z=0$ (b) splits into four filaments at $z=104$ and (c) coalesces at $z=221$, (d) reaching the solitonic equilibrium after many oscillations $(z=2800)$.

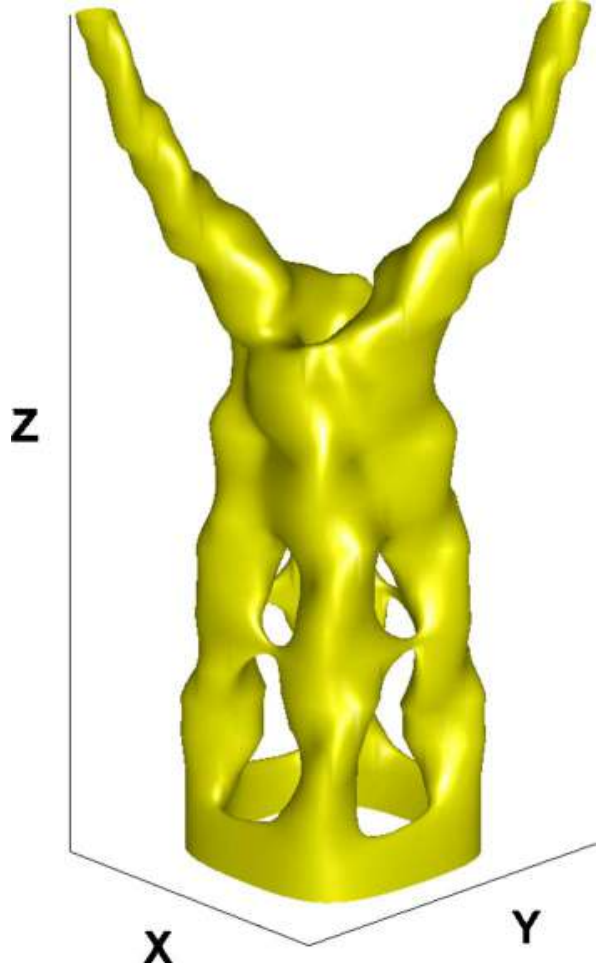

FIG. 7. (Color online) The ring splits and coalesces twice before breaking into two running away filaments (from $z=55$ till $z=650$ ).

be self-organized in narrow-gap semiconductors and can propagate as LOVS due to the synergy between the selffocusing cubic nonlinearity related to the nonparabolicity and the self-defusing quintic nonlinearity induced by nonequilibrium free carriers generated by two-photon absorption. The linear stability analysis and numerical simulations confirm the stability of powerful LOVS dominated by selfdefocusing and breaking into filaments of less powerful ones. Far from equilibrium, the richness of singular pulse dynamics seems to be the consequence of an interplay of two opposite tendencies: spreading filamentation induced by modulation instability and the attraction by equilibrium LOVS states. In order to have better look inside into such a complex behavior, GNLS is solved approximately using the variation method. A stability criterion is established giving the condition to self-organization into LOVS. Far from equilibrium, multiple filamentations can occur. A coalescence domain is established. For small amplitudes, filaments coalesce and split again. Powerful pulses with large amplitudes coalesce into stable vortex solitons, while powerless vortices reach the unstable equilibrium breaking into two filaments.

The vortex structure of the laser pulses contributes in general, if not to suppress the filamentation, at least to distribute filaments symmetrically around the singularity due to the conservation of the angular momentum. Therefore, a vortex structure prevents chaotic filamentation so often observed in powerful laser pulses without the singularity. Moreover, detailed studies of singular pulses evolution may contribute to an effective control of undesired filamentation by the choice of input parameters leading to the coalescence. 


\section{ACKNOWLEDGMENTS}

Work at the Institute of Physics is supported by the Ministry of Science of the Republic of Serbia under the Project No. OI 141031. This research has been in part supported by
French-Serbian cooperation, CNRS/MSCI Agreement No. 20504. The work of V.I.B. was supported by ISTC Project G-1366 and Georgian NSF grant project GNSF 195/07 (GNSF/ST07/4-191).
${ }^{1}$ D. Mihalache, D. Mazilu, F. Lederer, Y. V. Kartashov, L. C. Crasovan, L. Torner, and B. A. Malomed, Phys. Rev. Lett. 97, 073904 (2006).

${ }^{2}$ A. Desyatnikov, A. Maimistov, and B. A. Malomed, Phys. Rev. E 61, 3107 (2000).

${ }^{3}$ H. Michinel, J. Campo-Táboas, M. L. Quiroga-Teixeiro, J. R. Salgueiro, and R. García-Fernández, J. Opt. B: Quantum Semiclassical Opt. 3, 314 (2001).

${ }^{4}$ G. A. Swartzlander, Jr. and C. T. Law, Phys. Rev. Lett. 69, 2503 (1992); Yu. S. Kivshar and B. Luther-Davies, Phys. Rep. 298, 81 (1998).

${ }^{5}$ H. Michinel, J. R. Salgueiro, and M. J. Paz-Alonso, Phys. Rev. E 70, 066605 (2004).

${ }^{6}$ W. J. Firth and D. V. Skryabin, Phys. Rev. Lett. 79, 2450 (1997); D. V. Skryabin and W. J. Firth, Phys. Rev. E 58, 3916 (1998).

${ }^{7}$ M. S. Bigelow, P. Zerom, and R. W. Boyd, Phys. Rev. Lett. 92, 083902 (2004).

${ }^{8}$ M. Quiroga-Teixeiro and H. Michinel, J. Opt. Soc. Am. B 14, 2004 (1997).

${ }^{9}$ V. I. Berezhiani, V. Skarka, and N. B. Aleksić, Phys. Rev. E 64, 057601 (2001); V. Skarka, V. I. Berezhiani, and N. B. Aleksić, Phys. Lett. A 291, 124 (2001).

${ }^{10}$ Z. Birnbaum and B. A. Malomed, Physica D 237, 3252 (2008).

${ }^{11}$ V. Skarka, N. B. Aleksić, and V. I. Berezhiani, Phys. Lett. A 319, 317 (2003).

${ }^{12}$ V. Skarka and N. B. Aleksić, PIERS Online 4, 666 (2008).

${ }^{13}$ A. Vinçotte and L. Berge, Phys. Rev. Lett. 95, 193901 (2005).

${ }^{14}$ A. Vinçotte and L. Berge, Physica D 223, 163 (2006).

${ }^{15}$ D. Mihalache, D. Mazilu, B. A. Malomed, and F. Lederer, J. Opt. B: Quantum Semiclassical Opt. 6, S341 (2004).

${ }^{16}$ M. J. Paz-Alonso and H. Michinel, Phys. Rev. Lett. 94, 093901 (2005).
${ }^{17}$ A. A. Borshch, M. Brodin, and V. Volkov, Refractive Nonlinearity of Wide-Band Semiconductors and Applications, Laser Science and Technology (Harwood Academic Publishers, Chur, Switzerland, 1990), Vol. 9.

${ }^{18}$ O. Kane, J. Phys. Chem. Solids 1, 249 (1957); P. A. Wolff and G. E. Pearson, Phys. Rev. Lett. 17, 1015 (1966).

${ }^{19}$ N. Tzoar and J. I. Gersten, Phys. Rev. Lett. 26, 1634 (1971); Phys. Rev. B 4, 3540 (1971).

${ }^{20}$ P. K. Dubey and V. V. Paranjape, Phys. Rev. B 8, 1514 (1973).

${ }^{21}$ M. C. Downer, R. L. Fork, and C. V. Shank, J. Opt. Soc. Am. B 2, 595 (1985).

${ }^{22}$ V. I. Berezhiani and S. M. Mahajan, Phys. Rev. Lett. 73, 1837 (1994); Phys. Rev. B 55, 9247 (1997).

${ }^{23}$ V. I. Berezhiani, V. Skarka, and R. Miklaszewski, Phys. Rev. B 57, 6251 (1998).

${ }^{24}$ C. C. Wang and N. W. Ressler, Phys. Rev. 188, 1291 (1969).

${ }^{25}$ B. La Fontaine, F. Vidal, Z. Jiang, C. Y. Chien, D. Comtois, A. Desparois, T. W. Johnston, J.-C. Kieffer, and H. Pépin, Phys. Plasmas 6, 1615 (1999); N. Aközbek, C. M. Bowden, A. Talebpour, and S. L. Chin, Phys. Rev. E 61, 4540 (2000).

${ }^{26}$ A. Miller, A. Johnston, J. Dempsey, J. Smith, C. R. Pidgeon, and G. D. Holah, J. Phys. C 12, 4839 (1979).

${ }^{27}$ V. Skarka, V. I. Berezhiani, and R. Miklaszewski, Phys. Rev. E 56, 1080 (1997).

${ }^{28}$ V. E. Zakharov, V. V. Sobolev, and V. C. Synakh, Zh. Eksp. Teor. Fiz. 60, 136 (1971) [Sov. Phys. JETP 33, 77 (1971)].

${ }^{29}$ V. Skarka and N. B. Aleksić, Phys. Rev. Lett. 96, 013903 (2006); N. B. Aleksić, V. Skarka, D. V. Timotijević, and D. Gauthier, Phys. Rev. A 75, 061802(R) (2007).

${ }^{30}$ V. Skarka, D. V. Timotijević, and N. B. Aleksić, J. Opt. A, Pure Appl. Opt. 10, 075102 (2008). 